\title{
Comunicación corta
}

\section{Desprendimiento hemorrágico de la membrana de Descemet tras viscocanalostomía}

\author{
R. Gallego-Pinazo ${ }^{a, *}$, E. López-Sánchez ${ }^{b}$ y J. Marín-Montiel ${ }^{b}$ \\ ${ }^{a}$ Hospital Universitario La Fe, Valencia, España \\ ${ }^{\mathrm{b}}$ Hospital Arnau de Vilanova, Valencia, España
}

INFORMACIÓN DEL ARTÍCULO

Historia del artículo:

Recibido el 13 de noviembre de 2007

Aceptado el 26 de enero de 2010

\section{Palabras clave:}

Viscocanalostomía

Cirugía filtrante

Desprendimiento de la membrana

de Descemet

Descematopexia

SF6

\section{R E S U M E N}

Introducción: La viscocanalostomía constituye una alternativa en el tratamiento quirúrgico del glaucoma. Esta técnica no penetrante favorece el drenaje del acuoso a través del canal de Schlemm evitando la aparición de ampollas de filtración y de las patologías relacionadas con éstas. Las complicaciones asociadas a esta cirugía son escasas; una de ellas es el desprendimiento de la membrana de Descemet (DMD).

Caso clínico: Mujer de 64 años diagnosticada de glaucoma crónico de ángulo abierto cuyo ojo izquierdo fue intervenido mediante viscocanalostomía. En el posoperatorio inmediato apareció un DMD hemorrágico en el cuadrante temporal inferior. Tras adoptar inicialmente una actitud expectante, a los 15 días de la cirugía no se objetivó mejoría del DMD y se instauró un edema corneal que produjo disminución de la visión. Se procedió a la reaplicación quirúrgica del defecto mediante micropunción de la membrana de Descemet e inyección de SF6 en cámara anterior. Se consiguió una mejoría anatómica y funcional del cuadro.

Discusión: Pensamos que la salida del material viscoelástico al espacio supradescemético fue consecuencia de la alta presión en el proceso de inyección del hialuronato de alta densidad. El manejo de un DMD hemorrágico está condicionado fundamentalmente por su localización, tamaño y evolución. La actuación diferida mediante micropunción endotelial y posterior descematopexia por medio de inyección en cámara anterior de SF6 al 20\% resultó de utilidad para solucionar esta complicación.

(c) 2007 Sociedad Española de Oftalmología. Publicado por Elsevier España, S.L. Todos los derechos reservados.

\footnotetext{
*Autor para correspondencia.

Correo electrónico: robertogallego@comv.es (R. Gallego Pinazo).
} 


\section{Hemorrhagic Descemet's membrane detachment after viscocanalostomy}

\section{A B S T R A C T}

Keywords:

Viscocanalostomy

Filtering Surgery

Descemet's Membrane detachment

Descematopexy

SF6
Introduction: Viscocanalostomy is an option in the surgical treatment of glaucoma. This non-penetrating technique favours aqueous drainage through the Schlemm channel while avoiding filtering blebs and their related pathologies. Complications associated to this surgery are unusual, one of which is Decemet's membrane detachment (DMD)

Clinical case: A 64 year-old female diagnosed with open-angle chronic glaucoma, whose had undergone a viscocanalostomy of the left eye. In the immediate postoperative period we noticed a hemorrhagic DMD in the lower temporal quadrant. After adopting a wait and see attitude, we did not find improvement 15 days after surgery and corneal edema was established with vision decrease. We proceeded to a surgical reapplication by means of Descemet's membrane micropuncture and SF6 injection into the anterior chamber and achieving an anatomical and functional improvement

Discussion: We believe that the intrusion of viscoelastic material into the supradescemetic was a consequence of the high-pressure during the high-density hyaluronate injection. Hemorrhagic DMD management is mainly determined by its location, size and evolution. In our case, the posterior endothelial micropuncture and descematopexy by means of $20 \%$ SF6 injection into the anterior chamber was useful in resolving this complication

(c) 2007 Sociedad Española de Oftalmología. Published by Elsevier España, S.L.

All rights reserved.

\section{Introducción}

La viscocanalostomía es una alternativa en el tratamiento quirúrgico del glaucoma ${ }^{1}$. Esta técnica penetrante favorece el drenaje del acuoso a través del canal de Schlemm evitando la aparición de ampollas de filtración y de las complicaciones asociadas a éstas.

Son escasas las referencias de complicaciones secundarias a esta técnica quirúrgica ${ }^{2}$ y el número de publicaciones es aún más reducido en cuanto a la presencia de desprendimientos desceméticos como complicación de la viscocanalostomía, particularmente de las formas hemorrágicas ${ }^{3}$.

Presentamos un caso de un desprendimiento hemorrágico descemético por hialuronato sódico de alta densidad (Healon $\left.\mathrm{GV}^{\circledR}\right)$ que precisó de tratamiento quirúrgico para su drenaje.

\section{Caso clínico}

Mujer de 64 años diagnosticada de glaucoma crónico de ángulo abierto cuyo ojo izquierdo fue intervenido mediante viscocanalostomía. Su mejor agudeza visual corregida era de 0,7 y 0,8 en el ojo derecho (OD) y en el ojo izquierdo (OI) respectivamente. La presión intraocular (PIO) preoperatoria en el OI era de $26 \mathrm{mmHg}$ a pesar del tratamiento con la asociación de maleato de timolol y dorzolamida dos veces al día junto con latanoprost una vez al día. En el OD, con la misma pauta, la PIO era de $17 \mathrm{mmHg}$. Tanto en el estudio de las papilas ópticas como en el seguimiento del campo visual, se comprobó un deterioro progresivo en el OI.

Se decidió proceder a la realización de una viscocanalostomía según técnica habitual de Stegmann ${ }^{1}$. Realizamos la disección conjuntival con base en fórnix y tras la disección de un colgajo escleral que sobrepasó la línea de Schwalbe creamos un segundo flap escleral más profundo, que fue retirado, donde procedimos a localizar el canal de Schlemm y canalizarlo cuidadosamente hacia el interior inyectando el viscoelástico. Finalmente procedimos a suturar fuertemente el colgajo escleral superficial con nylon 10-0.

A las 24 horas de la intervención, la paciente presentaba un desprendimiento de la membrana de Descemet (DMD) en el cuadrante temporal inferior de 5,0 x 5,0 $\mathrm{mm}$. El espacio entre la membrana de Descemet y el estroma corneal presentaba un importante relleno hemático y por viscoelástico (figuras 1 y 2). La agudeza visual (AV) era en este

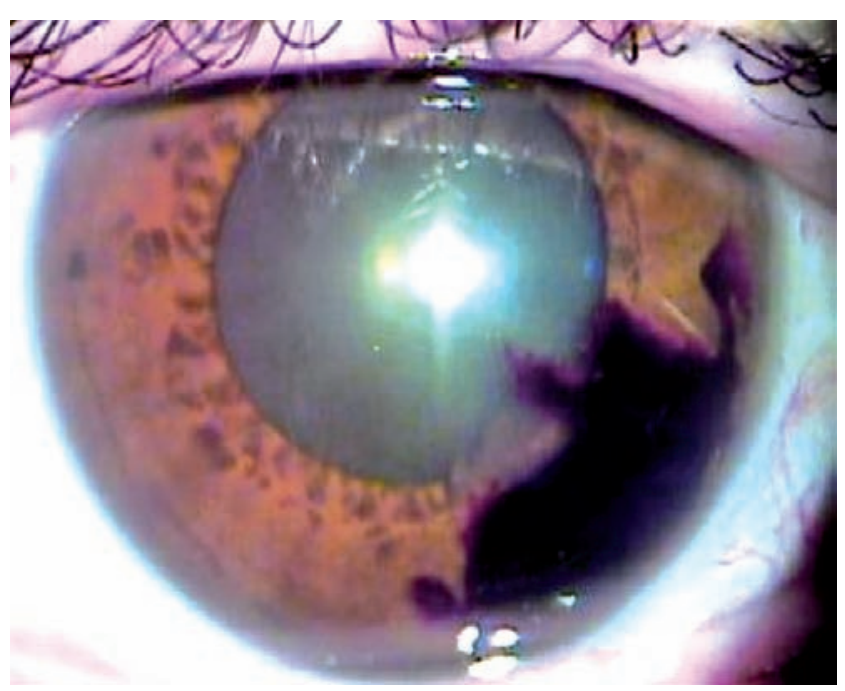

Figura 1 - Desprendimiento descemético hemorrágico en el cuadrante temporal inferior. 


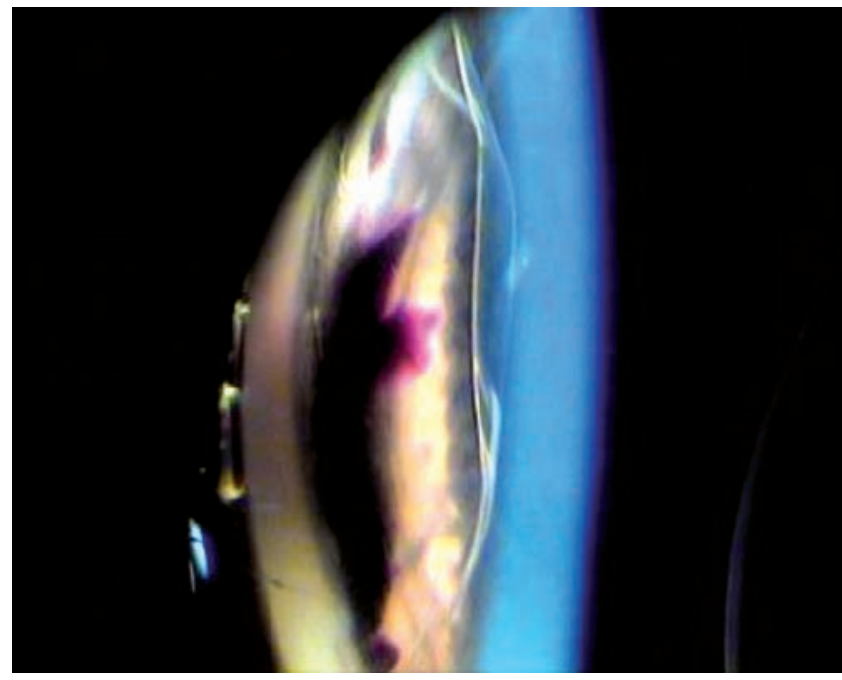

Figura 2 - Imagen gonioscópica en la que se aprecia la separación de la membrana de Descemet con inclusión del hialuronato sódico de alta densidad y la hemorragia a dicho nivel.

momento de 0,5 y su PIO $16 \mathrm{mmHg}$. Se pautó tratamiento con midriáticos (ciclopentolato) y esteroides tópicos (dexametasona) y se llevaron a cabo controles seriados mediante control de la AV, biomicroscopía, gonioscopía y toma de presión intraocular.

A las dos semanas de la intervención el estado de la inclusión corneal permanecía prácticamente inalterado, apreciándose además un edema corneal moderado que provocaba una disminución de la AV a 0,05 (fig. 3), por lo que se decidió la reaplicación quirúrgica haciendo uso de la siguiente técnica: con un miringotomo se realizó una rotura intencionada en la parte inferior del desprendimiento des-

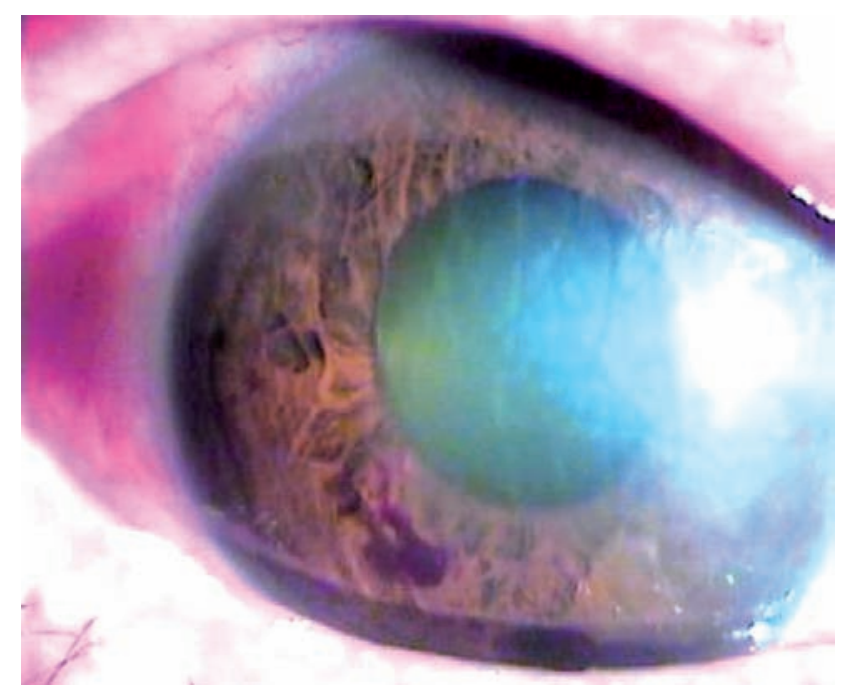

Figura 3 - A las dos semanas de la intervención el aspecto de la inclusión corneal permanecía prácticamente inalterado, apreciándose un edema corneal moderado que provocaba una disminución de la agudeza visual a 0,05.

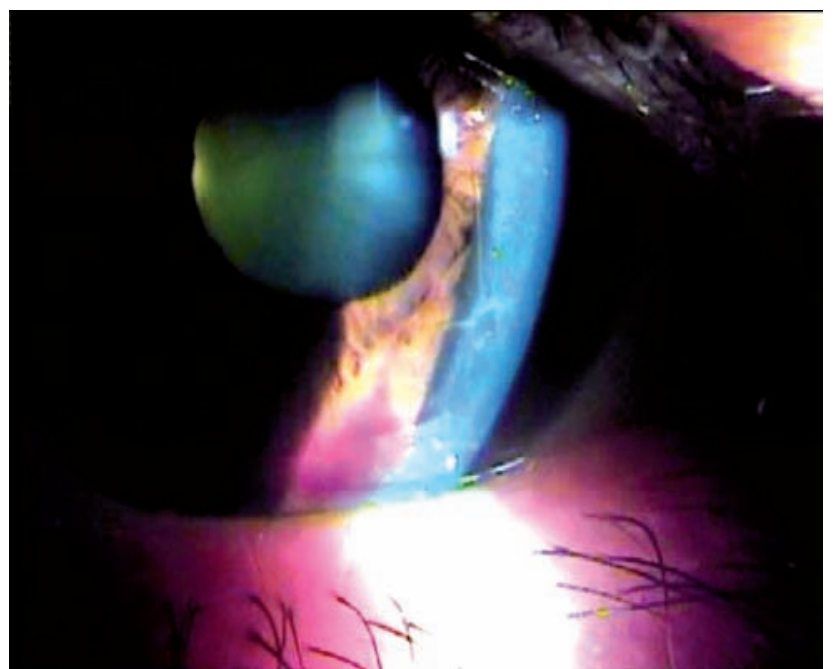

Figura 4 - Tras la descematopexia quirúrgica el edema corneal desapareció totalmente; solo se observa la presencia de pliegues desceméticos y algún pequeño resto hemático.

cemético y mediante la introducción repetida de aire y suero salino balanceado en la cámara anterior fue drenándose tanto el viscoelástico como el hematoma a través de la apertura quirúrgica descemética. Posteriormente se inyectó SF6 para mantener aplicado el endotelio corneal.

Una semana después de este procedimiento quedó completamente reaplicado el DMD, con pliegues radiados desde la zona afectada (fig. 4), pero con desaparición total del edema corneal, y recuperación de la AV a 0,8. Su PIO sin medicación fue de $16 \mathrm{mmHg}$.

\section{Discusión}

Nuestra impresión clínica es que la salida del material viscoelástico al espacio supradescemético fue consecuencia de la alta presión en el proceso de inyección del hialuronato de alta densidad. En este caso es particularmente llamativo el hecho de que el DMD se localizara únicamente en el cuadrante temporal inferior, con la membrana descemética perfectamente aplicada desde el punto de inyección a las XII horas y el comienzo del despegamiento, por lo que elaboramos la hipótesis de que el hialuronato se canalizó a través del Schlemm y en algún punto de su recorrido indujo una rotura en su pared con salida del material y posterior reflujo hemático.

Esta particularidad diferencia éste de la mayoría de los casos publicados ${ }^{2-5}$, en los que la disección de la Descemet se produce directamente desde el lugar de la inyección, supuestamente por un mecanismo de miss-direction del hialuronato con una participación mínima o nula del canal de Schlemm.

El manejo del DMD hemorrágico está condicionado fundamentalmente por su localización, tamaño y evolución. La mayoría de los autores abogan por el control médico durante el posoperatorio inmediato esperando la reabsorción de la 
hemorragia y la reaplicación endotelial espontánea, y reservan la intervención quirúrgica para cuando no se evidencie respuesta positiva ${ }^{2}$.

Algunos artículos nos muestran resultados muy positivos tras la reaplicación descemética con SF6 20\% . Otras alternativas para la pexia serían la inyección de aire o viscoelástico en la cámara anterior ${ }^{2}$ Estas técnicas tienen la desventaja de la rápida reabsorción del aire o el aumento transitorio de la PIO que producen los viscoelásticos. Otro método más complejo sería la utilización de suturas transcorneales junto con la inyección de aire ${ }^{5}$.

Nosotros optamos por la actuación diferida mediante micropunción endotelial para facilitar el drenaje del hialuronato encapsulado bajo la Descemet y posterior descematopexia a través de la inyección en cámara anterior de SF6 al $20 \%$.

\section{B I B L I O G R A F Í A}

1. Stegmann R, Pienaar A, Miller D. Viscocanalostomy for openangle glaucoma in black African patients. J Cataract Refract Surg. 1999;25:316-22.

2. Lüke C, Dietlein T, Jacobi P, Konen W, Krieglstein GK. Intracorneal inclusion of high-molecular-weight sodium hyaluronate following detachment of Descemet's membrane during viscocanalostomy. Cornea. 2000;19:556-7.

3. Kozobolis VP, Christodoulakis EV, Siganos CS, Pallikaris IG. Hemorrhagic Descemet's membrane detachment as a complication of deep sclerectomy: a case report. J Glaucoma. 2001;10:497-500.

4. Ocakoglu O, Ustundag C, Devranoglu K, Yildrim R, Ozkan S. Repair of Descemet's membrane detachment after viscocanalostomy. J Cataract Refract Surg. 2002;28:1703-6.

5. Amaral CE, Palay DA. Technique for repair of Descemet membrane detachment. Am J Ophthalmol. 1999;127:88-90. 